\title{
Increased Frequency and Voltage Interactions Affecting Frequency and Transient Stability in Networks with Large Penetration of Renewable Generation
}

\author{
Atia Adrees \& \\ Jovica V. Milanović \\ School of Electrical and Electronic \\ Engineering \\ The University of Manchester, UK
}

\author{
Panagiotis N. Papadopoulos \\ Department of Electronic \& Electrical \\ Engineering \\ University of Strathclyde \\ UK
}

\author{
Pierluigi Mancarella \\ Department of Electrical \& Electronic \\ Engineering \\ The University of Melbourne \\ Australia
}

\begin{abstract}
The paper investigates the frequency and voltage dynamics and stability in power systems with increased penetration of inverter-based renewable energy sources (RES). The case studies presented within the paper show that the frequency dynamics (frequency nadir and rate of change of frequency) is not only affected by the decrease in system inertia but also by increasing frequency/voltage interactions when the proportion of RES exceeds that of synchronous generation. Furthermore, the critical fault clearing Time (CCT) analysis for transient stability indicates that RES fault ride through (FRT) and their settings can have a significant impact on the nearby generators. The studies also demonstrate that voltage and frequency interactions can be reduced and transient stability of synchronous generators improved by applying dynamic voltage support in weak areas of the system.
\end{abstract}

Index Terms- Frequency stability, System inertia, Renewable energy sources, Transient stability, Low-inertia power systems

\section{INTRODUCTION}

$\mathrm{T}$ HE security of future power systems will be challenged by the increasing penetration of inverter-based renewable energy sources (RES). The large-scale integration of RES into electricity grids have an impact on power flows as well as voltage and frequency control, which change the dynamic behavior of the system considerably and in a complex manner.

Inverters decouple a rotating mass from the rest of the system even though the prime mover behind the inverter may have some inertia (as in the case of wind turbines), hence, there is no natural inertia contributed by an inverter connected rotating mass [1, 2]. By displacing synchronous generation (SG), high penetration of RES thus reduces the natural inertia of the system and influences the rate of change

This work is supported by the UK Engineering\&Physical Science Research Council (EPSRC)-China collaborative project "RESTORES", the EPSRC-India collaborative project "ACCEPT", and the EPSRC project "MY-STORE". of frequency (RoCoF) and the frequency nadir. The high RoCoF also means the large transient displacement of voltage angles in different zones of the grid that may induce pole slipping of SGs and network splitting, possibly causing distance protection tripping. One solution could be to deploy inertia potentially available from RES, for example from wind generation. However, to do so a significantly oversized inverter may be necessary due to inverter current and voltage limitations [3]. Furthermore, the "synthetic "inertia provided by RES cannot exactly match the behavior of the natural inertia of SG, due to measurement and control action delays[3].

Many studies have been performed by using a traditional system equivalent model approach where the collective performance of all generators is assessed [4-6]. Analytical methods that use state space models linearized around the operating point were also used to determine the optimal amount and location of inertia [7]. In these analysis converter connected renewable generators are modelled as generic power sources with specified inertia and damping, with an underlying assumption that those could be synthesized using a power electronics inverter [7]. A framework for assessing renewable integration limits with respect to frequency adequacy, using a simplified four-area system of Australia, was proposed in [8]. The authors proposed a framework to analyze the impact of increased penetration of RES on grid frequency taking into account the stochastic and intermittent patterns of RES [9]. The proposed methodology successfully identified the critical penetration levels and critical inertia of the system. Recently there has been significant interest in incorporating post-contingency requirements in economic dispatch and unit commitment models $[10,11]$. Minimum inertia requirements are included in economic dispatch in [12], and RoCoF and frequency nadir constraints are added 
into unit commitment and economic dispatch in $[10,13,14]$.

Synchronous generators are also the backbone of voltage control in the electric power networks. The voltage support provided by SG in addition to voltage support provided by conventional capacitors as well as static devices (e.g., SVCs and STATCOMs) is particularly important as it does not lose effectiveness when the terminal voltage decreases, as is the case with other devices. Besides being auto-stabilizing, the response of an SG to an emergency reactive power requirement is much faster and more accurate than that of static reactive sources [15]. Furthermore, inverters lack inductive characteristics that are associated with rotating machines. Even though they can contribute to short circuit current, this contribution is typically limited to 1 pu provided that all the active power is reduced to zero, and all the current which is able to flow through the valves is turned into reactive power [2].

Though frequency and voltage phenomena are intrinsically linked, as it can also be appreciated from the above considerations, they are typically studied independently in the design and operation of electrical networks. However, the high penetration of RES increases these interactions which may strongly affect frequency dynamics and large disturbance rotor angle stability.

This paper investigates the effect of frequency and voltage interactions on frequency dynamics and large disturbance rotor angle stability of the network. The performed analysis indicates that when the proportion of RES becomes greater than SGs in an individual area of the network, frequency dynamics are not only affected by the decrease in inertia but also due to increased frequency and voltage interactions. The performed analysis shows clearly that the impact of these interactions can be minimized by introducing dynamic voltage support in the weak area of the network that improves grid frequency response and also large disturbance stability of SGs.

\section{TEST NETWORK}

To investigate the interactions between frequency and voltage due to an increased penetration of inverter connected RES, a well-known small test system (adapted from [3] where system data is presented) is selected. A small test network is chosen to facilitate easy explanation.

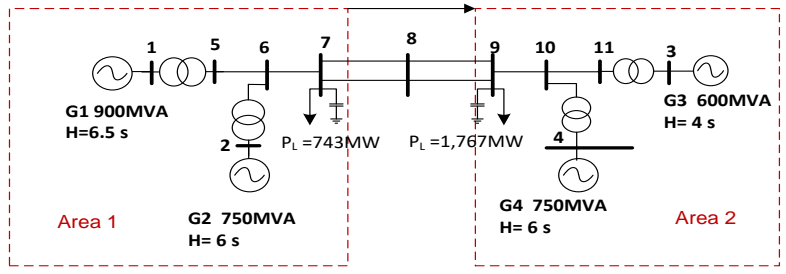

Fig. 1: Four Machine Network

The network consists of two areas, with G1 and G2 connected in area 1, and G3 and G4 in area 2. The active power is flowing from area 1 to area 2 . All generators are equipped with the fast-acting IEEE-ST1A exciter. Generators G2 and G4 are represented by three parallel machines of the same size and inertia constant. All generators are modeled with a $6^{\text {th }}$ order model. Lines are represented by $\pi$ equivalent models. Each load in the network is modeled as a combination of constant impedance and constant power load. The governors are blocked to isolate the effect of a decrease in inertia.

RES are represented by a Type 4 WTG model for system stability. Type 4 WTG are full converter connected (FCC) generators. All generators connected to the grid through full converter interface like PVs can be represented by the Type 4 WTG model for system stability studies [16]. The modelling approach is similar to Type 4 models in [17]. Current controller, PQ controller and over frequency power reduction control of the converter are also included in the model. The test network is implemented in DIgSILENT PowerFactory.

\section{A. Case studies}

The following case studies are developed to examine the extent of frequency and voltage interactions on frequency dynamics and large disturbance rotor angle stability.

CS I: The standard network

CS II: One out of three 250 MVA units of G2 is replaced by a full converter connected RES of the same size.

CS III: Two out of three 250 MVA units of G2 are replaced by a full converter connected RES of the same size.

CS IV: Entire G2 is replaced by FCC RES of 750 MVA.

CS V: One 250 MVA unit of G4 is replaced by FCC RES.

CS VI: Two units (500 MVA) of G4 are replaced by FCC RES.

CS VII: Entire G4 (750 MVA) is replaced by a FCC RES.

Different disturbances are applied to stress the system and analyse its dynamic behavior, as discussed below.

\section{RESULTS AND ANALYSIS}

In these studies, the original test network without RES (CS I) is compared with the cases where FCC generation incrementally displaces SG but in different areas, namely, CS II-IV for Area 1 and CS V-VII for Area 2. The summary of the resulting inertia constants and RES penetration levels per area and for the whole network is given in Table I and Table II.

TABLE I: WHOLE NETWORK $(\mathrm{H})$ AND AREA $\left(H_{i}\right)$ INERTIA FOR CS I-CS VII

\begin{tabular}{ccccccccc}
\hline \hline \multirow{2}{*}{$\begin{array}{c}\text { Inertia } \\
\text { constant }\end{array}$} & \multirow{2}{*}{ No RES } & \multicolumn{3}{c}{ Replacement of G2 } & \multicolumn{3}{c}{ Replacement of G4 } \\
\cline { 3 - 10 } & CS I & $\begin{array}{l}\text { 1 unit } \\
\text { CS II }\end{array}$ & $\begin{array}{c}\text { 2 units } \\
\text { CS III }\end{array}$ & $\begin{array}{l}\text { Full G2 } \\
\text { CS IV }\end{array}$ & $\begin{array}{c}\text { 1 unit } \\
\text { CS V }\end{array}$ & $\begin{array}{c}\text { 2 units } \\
\text { CS VI }\end{array}$ & $\begin{array}{c}\text { Full G4 } \\
\text { CS VII }\end{array}$ \\
\hline Area 1 & 6.27 & 5.36 & 4.45 & 3.54 & 6.27 & 6.27 & 6.27 \\
\hline Area 2 & 5.11 & 5.11 & 5.11 & 5.11 & 4 & 2.88 & 1.77 \\
\hline Network & 5.75 & 5.25 & 4.75 & 4.25 & 5.25 & 4.75 & 4.25 \\
\hline \hline
\end{tabular}

It can be observed that as the share of RES gradually increases to $45 \%$ (Table II) in area 1, the inertia constant of area 1 decreases from $6.27 \mathrm{~s}$ to $3.54 \mathrm{~s}$. Similarly, the displacement of SG of the same size increases the penetration 
of RES to $55 \%$ in area 2 and the inertia constant of area 2 drops to $1.77 \mathrm{~s}$. Although the displacement of G4 units leads to a greater decrease in the inertia constant of area 2, the average inertia of the system stays the same whether SG is disconnected in area 1 or in area 2.

TABLE II: PENETRATION LEVELS OF RES FOR CS I-CS VII

\begin{tabular}{cccccccc}
\hline \hline & \multirow{2}{*}{ \% RES } & No RES & \multicolumn{3}{c}{ Replacement of G2 } & \multicolumn{3}{c}{ Replacement of G4 } \\
\cline { 2 - 9 } & CS I & $\begin{array}{l}\text { 1 unit } \\
\text { CS II }\end{array}$ & $\begin{array}{c}\text { 2 units } \\
\text { CS III }\end{array}$ & $\begin{array}{l}\text { Full G2 } \\
\text { CS IV }\end{array}$ & $\begin{array}{l}\text { 1 unit } \\
\text { CS V }\end{array}$ & $\begin{array}{c}\text { 2 units } \\
\text { CS VI }\end{array}$ & $\begin{array}{c}\text { Full G4 } \\
\text { CS VII }\end{array}$ \\
\hline Area 1 & $0 \%$ & $15 \%$ & $30 \%$ & $45 \%$ & $0 \%$ & $0 \%$ & $0 \%$ \\
\hline Area 2 & $0 \%$ & $0 \%$ & $0 \%$ & $0 \%$ & $18 \%$ & $37 \%$ & $55 \%$ \\
\hline Network & $0 \%$ & $8 \%$ & $16 \%$ & $25 \%$ & $8 \%$ & $16 \%$ & $33 \%$ \\
\hline \hline
\end{tabular}

\section{B. Active power disturbance at bus 7}

Fig. 2 shows the grid frequency response at bus7 following an active power disturbance (increase in demand) when RES gradually displaces SGs in Area I. The active power disturbance is introduced at bus 7 and it is $5 \%$ of the total demand in the network. The frequency nadir following the active power disturbance in the system without RES is $49.63 \mathrm{~Hz}$ which drops to $49.5 \mathrm{~Hz}$ when penetration of RES increases to $45 \%$ following the displacement of entire G2. The increase in RoCoF is also small; it grows from $0.2 \mathrm{~Hz} / \mathrm{s}$ to $0.22 \mathrm{~Hz} / \mathrm{s}$.

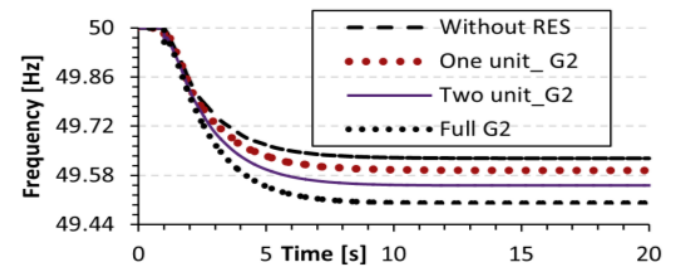

Fig. 2: Grid frequency response following an active power disturbance at bus 9; CS I-CS V

The RoCoF and the drop in the frequency stay the same whether one or two units of SGs are disconnected in area 1 or area 2. However, when the entire G4 is replaced by RES, frequency and voltage interactions in the network become very strong, changing frequency dynamics significantly (Fig. 3). It can be seen that following the same active power disturbance the frequency of each generator does not drop, but instead increases initially (each generator in fact accelerates). The generator G3 first experiences a positive RoCoF of $1 \mathrm{~Hz} / \mathrm{s}$, and then a negative $\mathrm{RoCoF}$ of $0.9 \mathrm{~Hz} / \mathrm{s}$. The frequency drops to $49.3 \mathrm{~Hz}$ in area 2 , and $49.5 \mathrm{~Hz}$ in area 1. This can be explained by looking at the voltage of the buses connecting the two areas through tie-lines.

Following the increase in the load at bus7, the voltage at bus 7 and bus 9 drops to $0.7 \mathrm{pu}$ and 0.6 pu respectively (Fig. 4) reducing the power flow through the tie-lines. Power is flowing from area 1 to area 2 . Hence, SGs in area 1 accelerate. The frequency in area 1 increases. The power flow from G3 to bus 9 is also decreased due to the considerable voltage drop at bus 9, hence, G3, also accelerates. RES reduce their active power to provide reactive power (Fig. 5). Then voltages at bus 7 and bus 9 start to recover. The reduction in RES power appears as an active power disturbance, with the generator G3 being the nearest and "lightest" generator. Therefore, it experiences a higher RoCoF of $0.9 \mathrm{~Hz} / \mathrm{s}$, while the frequency drops to $49.3 \mathrm{~Hz}$ in area 2 and 49.5 in area 1 . At that point, voltage recovers to the nominal value, and RES increases their active power. These frequency and voltage oscillations last for seconds.

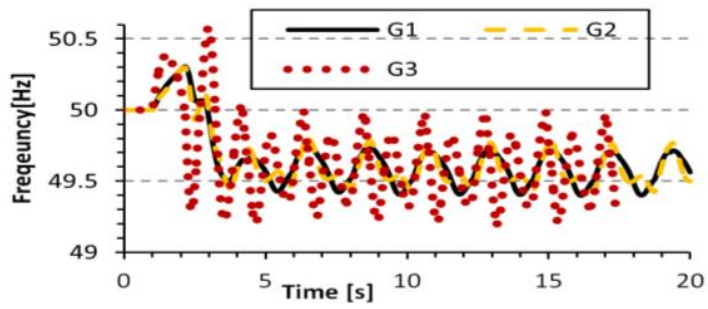

Fig. 3: Frequency response of G1, G2, and G3 following an active power disturbance at bus 7; CS VII

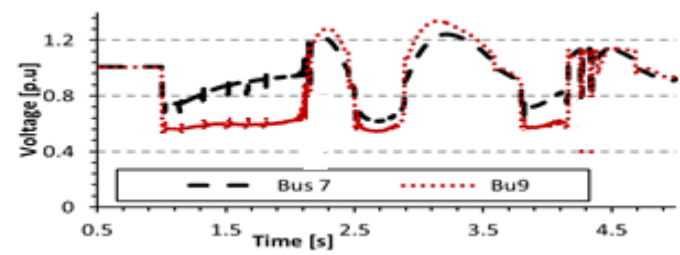

Fig. 4: Voltage profiles at bus 7 and bus 9 following the active power disturbance

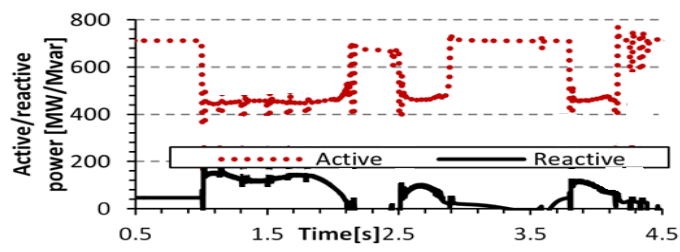

Fig. 5: Active and reactive power of RES

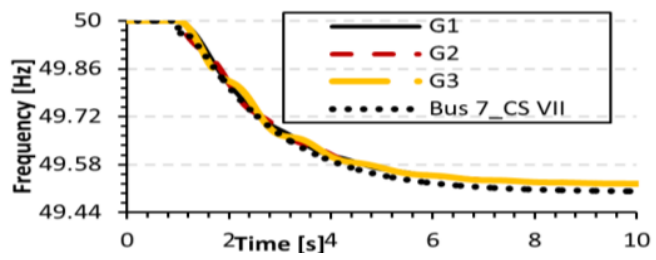

Fig. 6: Impact of dynamic voltage support on grid frequency response

To help reduce the impact of these frequency and voltage interactions, dynamic voltage support can be used. Dynamic voltage support can help to recover and maintain the voltage which in turn will support the recovery of the frequency. In the standard network, there is static reactive power support provided by capacitors at bus 7 and bus 9. To illustrate the effectiveness of dynamic voltage support, the 200 Mvar capacitors at bus 9 are disconnected, and a synchronous condenser of 100 Mvar is connected. Fig. 6 shows the frequency response of the system following the same active power disturbance when the entire G4 is replaced by RES; i.e., the system is operating with $55 \%$ penetration of RES in area 2. It can be seen that frequencies of SGs in both areas are 
the same and frequency response is very similar as it was when the entire G2 was disconnected.

\section{Three phase fault at bus 7}

To investigate further these frequency and voltage interactions in the network, CS I -CS VII are repeated by introducing a $70 \mathrm{~ms}$ self-clearing three-phase fault at bus 7 . Fig. 7 shows the frequency response of generator G3 following the three-phase fault without RES, replacement of one unit, two units and the entire G4 with RES, respectively. Generator G3 is the lightest generator in the network. Therefore, its frequency excursions are presented. Frequencies of all other generators show very similar but less pronounced behavior.

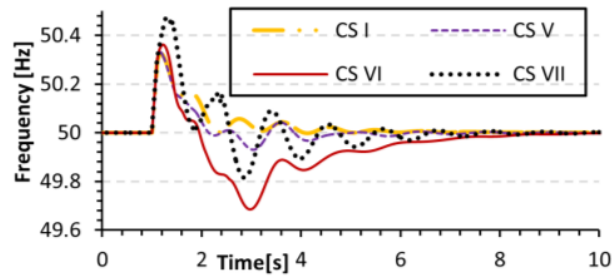

Fig. 7: Frequency response of G3 following a three-phase fault at bus 7 for gradual displacement of SGs in area 2, CS I and CS V-CS VII

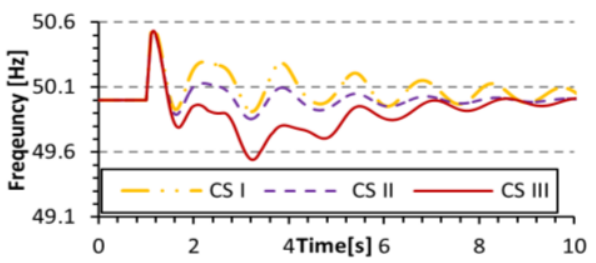

Fig. 8: Frequency response of G3 following a three-phase fault at bus 7 for gradual displacement of SGs in area 1, CS I, CS II-CS III

It can be seen that the increase in the initial peak of the frequency of the generator, following the fault, is negligible as penetration of RES increases (inertia of area 2 reduces). However, when two units of G4 are replaced by RES, a large dip in all generator frequency is observed. The dip in the frequency increases from $49.95 \mathrm{~Hz}$ to $49.7 \mathrm{~Hz}$. This change in the dynamic behaviour of the system can be explained by looking at the active powers of SGs and RES.

Following the three-phase fault at bus 7, the active power of each generator drops and generators' rotors accelerate. As the fault is cleared, active power increases and generators' rotors start to decelerate. This deceleration corresponds to the dip in their frequencies. The deceleration area is increased due to fault ride mode of RES. The voltage deadband of the RES controller is set in this study to $0.1 \mathrm{pu}$. After the fault is cleared, the voltage at bus 4 where RES is connected becomes higher than $1.1 \mathrm{pu}$ due to the assumed reactive power support from RES, and the active power of RES drops from 460MW to 190MW. This appears as a loss of generation in the network, resulting in SGs having to increase their active power and hence a bigger dip in the frequency of all generators. When the entire G4 is disconnected, the voltage drop at bus 9 becomes higher than in the previous case, and the reactive power support provided by RES does not cause overvoltage and thus a consequential reduction in active power support by RES. Hence, the large dip in generator frequency is not observed. On the other hand, the disconnection of the entire G4 leads to an increase in the peak of frequency of the generator.

Fig. 8 shows the frequency response of G3 when SGs in area 1 are displaced (CS II-CS IV). It can be seen that the frequency response is very similar when two units of G2 are removed, and the dip in the frequency is again increased. However, for the full disconnection of G2, G3 becomes unstable. This is due to RES controls. In fact, when their settings are changed, i.e., the voltage dead band is enlarged, the system becomes stable. If the fault is introduced at bus 9 for the full disconnection of G4, G2 becomes unstable and cannot be stabilized by changing the setting of RES controls.

It should be emphasized that the interactions between voltage and frequency, in addition to the RES penetration level, are strongly influenced by the type of controllers applied and controller settings of RES.

\section{EFFECT OF VOLTAGE FREQUENCY INTERACTIONS ON TRANSIENT STABILITY}

The Critical Clearing Time (CCT) has been widely used as an index to assess system transient stability, and thus we also use it in this study. The CCT is determined for case studies CS1-CS7 after introducing three-phase self-clearing faults at Bus 7 and Bus 9 .

The CCT for faults at Bus 9 is much smaller than that of Bus 7 for all cases. In general, as generators are disconnected (either G4 or G2), the CCT increases for a fault at Bus 7 while it decreases for a fault at Bus 9. This indicates that there is a positive effect on transient stability by the fault ride through (FRT) capability of RES for faults that are less severe, i.e., further from the critical area of the network. Furthermore, in the standard network as well as in the cases when the RES is connected close to G2, generators G3 and G4 tend to go unstable together. This indicates that area 2, power importing area, is the weaker area of the network. In cases when one and two units of G4 are replaced with RES, generator G3 is the first generator to exhibit instability. This indicates local support to generator G4 from FRT control of RES, when units within G4 are disconnected.

TABLE III: CCTS FOR FAULTS AT BUS7 AND Bus9

\begin{tabular}{cccc}
\hline & & \multicolumn{2}{c}{ CCT [s] } \\
\hline Standard network & & Bus7 & Bus9 \\
\hline \multirow{2}{*}{ One unit } & $\mathrm{G} 4$ & 0.63 & 0.26 \\
\cline { 2 - 4 } & $\mathrm{G} 2$ & 0.73 & 0.23 \\
\hline \multirow{2}{*}{ Two units } & $\mathrm{G} 4$ & 1.08 & 0.25 \\
\cline { 2 - 4 } & $\mathrm{G} 2$ & 0.98 & 0.24 \\
\hline \multirow{2}{*}{ Entire } & $\mathrm{G} 4$ & 0.34 & - \\
\cline { 2 - 4 } & $\mathrm{G} 2$ & - & 0.13 \\
\hline
\end{tabular}

When the faults are close to the critical generators G3 and G4 (bus 9), the disconnection of generators leads to a 
decrease in CCT. This behavior is much more prominent when the disconnection of synchronous generation occurs in the critical area of the network (area 2). Further inertia reduction inside a weak area causes a more significant decrease in the CCT. On the other hand, when inertia is reduced in the stronger area of the network (far from the generators causing the instabilities), the reduction in CCT is lower. Generator G3 becomes unstable when entire G4 is replaced by RES and fault is introduced at bus9. Similarly, G3 becomes unstable when entire G2 is replaced with RES and the fault is introduced at bus7.

In the simplest form (neglecting the damping constant), we can use the classical swing equation (1) to represent a generator's dynamics. Neglecting changes in the pre-fault operating conditions, the effect of RES on system stability has to do with i) inertia of the system being reduced and ii) $P_{e}$ being actively affected by RES controllers in a non-linear manner. (In the case of swing equation representing the equivalent generator, the mechanical power also changes for disconnection of individual units forming the equivalent generator under analysis.) While the effect of reduction in system inertia $H$ is easier to account for, it is not straightforward to analytically represent the effect of RES controllers on $P_{e}$ of generators, during and after disturbances. In general, the effect of RES control, assuming FRT capability, is to reduce their active power output and increase reactive power output to support the voltage. This discrete control action has an immediate effect on nearby generator power $P_{e}$ during and after the fault, which in turn affects the accelerating/decelerating areas and hence the overall stability of the generator. Coupling the effect of RES on $P_{e}$ with the reduced inertia in the system makes the effect on acceleration/deceleration of individual generators even more prominent.

$$
\frac{d \omega}{d t}=\frac{\omega_{0}}{2 H}\left(P_{m}-P_{e}\right)
$$

It is, therefore, important to identify critical areas/generators in the system and appropriately model disconnection and de-loading of SG. In particular, disconnecting generators in the stronger area of the network and de-loading generators in the weaker area can maintain or even improve the transient stability of a system.

CS IV and CS VII (Entire disconnection of G4 and G2) are repeated by installing a synchronous condenser at bus 9 . The CCT for CS IV becomes $0.14 \mathrm{~s}$ for a fault at bus 9 , and it became 0.63 for CS VII at bus 7. As expected, dynamic voltage support in weak areas of the network can support dynamic behaviour deterioration due to RES.

\section{CONCLUSIONS}

Currently, frequency and voltage events are considered separately and not combined as considered in this work.

The case studies presented within the paper show clearly that when the penetration of RES dominates synchronous generation in the weak area of the network the frequency dynamics, frequency nadir, and $\mathrm{RoCoF}$ can be strongly affected by voltage and frequency interactions. The impact of these interactions can be considerably reduced by identifying weak areas/buses in the system and providing dynamic voltage support. Control settings and RES location can also affect large disturbance rotor angle stability considerably in a weak area of the network. Dynamic voltage support in weak areas of the system reduces voltage and frequency interactions and also improves large disturbance rotor angle stability.

\section{REFERENCES}

[1] N. W. Miller et al., "Frequency response of California and WECC under high wind and solar conditions," in IEEE Power and Energy Society General Meeting, 2012.

[2] R.-C. S. P. D. S. Group, "Frequency Stability Evaluation Criteria for the Synchronous Zone of Continental Europe - Requirements and impacting factors -," REE, Terna, TransnetBW, 50HertzTransmission, RTE, Swissgrid and Energinet.dk.March 2016.

[3] S. M. Villanueva, "JWG C4/C6.35/CIRED Modelling and dynamic performance of inverter," CIGRE/CIRED2017.

[4] P.Kundur, Power System Stability \& Control. New York, London: McGraw Hill, 1994.

[5] A. Ulbig, et al, "Analyzing Rotational Inertia, Grid Topology and their Role for Power System Stability," IFAC-PapersOnLine, vol. 48, pp. 541-547, 2015.

[6] A. Ulbig et al., "Impact of Low Rotational Inertia on Power System Stability and Operation," IFAC Proceedings Volumes, vol. 47, pp. 7290-7297, 2014.

[7] B. K. Poolla, S. Bolognani, and F. Dörfler, "Optimal Placement of Virtual Inertia in Power Grids," IEEE Transactions on Automatic Control, vol. 62, pp. 6209-6220, 2017.

[8] A. S. Ahmadyar et al., "A Framework for Assessing Renewable Integration Limits with Respect to Frequency Performance," IEEE Transactions on Power Systems, 2017.

[9] A. Adrees, P. N. Papadopoulos, and J. V. Milanovic, "A framework to assess the effect of reduction in inertia on system frequency response," in Power and Energy Society General Meeting 2016.

[10] F. Teng, V. Trovato, and G. Strbac, "Stochastic scheduling with inertia-dependent fast frequency response requirements," IEEE Transactions on Power Systems, vol. 31, pp. 1557-1566, 2016.

[11] Y. Y. Lee and R. Baldick, "A Frequency-Constrained Stochastic Economic Dispatch Model," IEEE Transactions on Power Systems, vol. 28, pp. 2301-2312, 2013.

[12] H. Gu et al., "Minimum Synchronous Inertia Requirement of Renewable Power Systems," IEEE Transactions on Power Systems, 2017.

[13] R. Doherty, G. Lalor, and M. O'Malley, "Frequency control in competitive electricity market dispatch," IEEE Transactions on Power Systems, vol. 20, pp. 1588-1596, 2005.

[14] L. Zhang et al, "System-Level Operational and Adequacy Impact Assessment of Photovoltaic and Distributed Energy Storage, with Consideration of Inertial Constraints, Dynamic Reserve and Interconnection Flexibility," Energies, vol. 10, p. 989, 2017.

[15] O. A. Mousavi and R. Cherkaoui, "Literature survey on fundamental issues of voltage and reactive power control," Ecole Polytechnique Fédérale de Lausanne: Lausanne, Switzerland, 2011.

[16] W. R. E. M. T. Force, "WECC wind plant dynamic modeling guidelines," Western Electricity Coordinating Council Modeling and Validation Work Group, vol. 17, 2014.

[17] P. Sorensen, B. Andresen, J. Fortmann, and P. Pourbeik, "Modular structure of wind turbine models in IEC 61400-27-1," in IEEE Power and Energy Society General Meeting, 2013. 